\title{
PWR-DEPRESSURIZATION AND ITS HYDRAULIC ANALOGY
}

\author{
F. KEDZIUR, N. MOUSSIOPOULOS, U. SCHUMANN, K. STÖLTING \\ Kernforschungszentrum Karlsruhe, Institut für Reaktorentwicklung, \\ Postfach 3640, D-7500 Karlsruhe, Fed. Rep. of Germany
}

Received 9 November 1977

\begin{abstract}
The aim of this work is to provide an experimental verification of codes to be used for numerical simulation of the depressurization process in a pressurized water reactor (PWR) in case of a hypothetical blowdown accident. Here, the downcomer of a PWR is approximately treated as a two-dimensional plane region (unwrapped). The flow is simulated experimentally using the hydraulic analogy of free surface waves in a shallow water bed and compared to numerical simulations for the analogous flow of an ideal gas with an isentropic coefficient equal to two. The differences between the results of both models are less than $10 \%$.

Also reported are results of a similar study for a one-dimensional case (depressurization. in a pipe). The depressurization process can be subdivided into three phases: (1) Fluid still at rest, smooth depressurization wave, pressure drops, (2) Fluid accelerates, pressure recovers near the outlet (in 2-dim. case), (3) Quasi-steady flow, oscillations (in 2-dim. case). It is suggested that the results serve as a bench-mark for blow-down accident analy sis codes.
\end{abstract}

\section{Introduction}

For analysis of the dynamic loadings of the reactor vessel internals within a PWR vessel during a hypothetical blowdown accident, an extensive experimental and analytical program has been set up in which the former HDR-reactor is used as a test facility $[1,2]$. Of particular interest are the resultant flow velocity and pressure fields in the downcomer which is the annular gap between the vessel wall and the core barrel. Because the gap is narrow (150 $\mathrm{mm}$ ) compared to the vessel radius $(1480 \mathrm{~mm})$, two-dimensional effects are dominant so that the downcomer can be approximated as a plane flow region in the azimuthal $(x)$ and axial $(y)$ directions as shown in fig. 1. Because of symmetry only a $180^{\circ}$ section of the downcomer needs to be considered. The pipe in which the break is assumed is turned into the plane and set to zero length so that the break area is represented by a simple gap in the boundary. In this geometrical model the flow field has been analyzed numerically by means of the code YAQUIR [3] which integrates the Navier-Stokes equations using the implicit finite difference scheme developed by Hirt et al. [4].

In order to justify the application of computer codes like YAQUIR, DAPSY [5], WHAM [6], LECK [7], PWINCAD [8] or SOLA-FLX [9] to the problems considered here, these codes have to be verified experimentally. One way of verification is to compare computed results with experiments like those under preparation at the HDR which are as close as possible

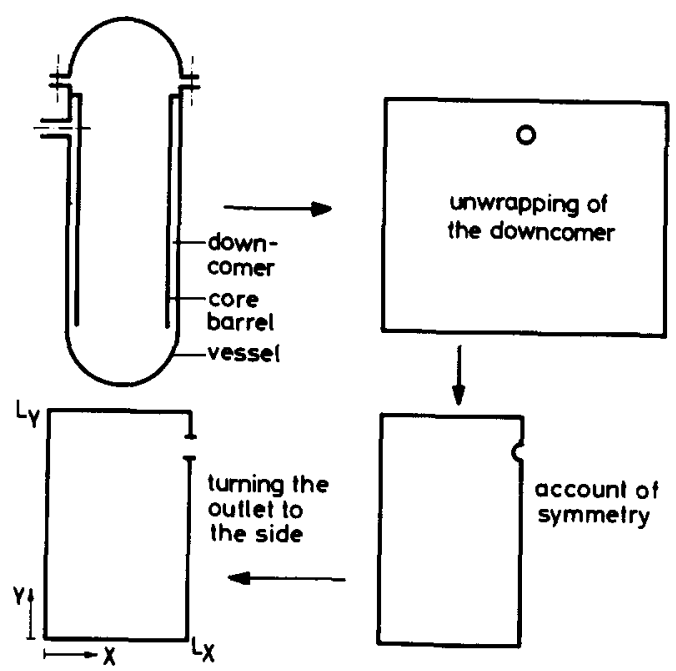

Fig. 1. Abstraction process of the geometrical model. 
to the real world problems. However, because such experiments envolve a tremendous financial engagement, only very few experimental data of this type are available and methods for fast and cheap verification of the numerical methods are urgently needed. Therefore, we have studied the flow problem experimentally by means of the well-known [10-12] hydraulic analogy in a shallow water bed $[13,14]$. Very appealing properties of such an experiment are its simplicity, its cheapness and the "visibility" of pressure, density, etc. The analogy allows one to study two-dimensional ideal gas-flow problems within complicated geometrical boundaries.

The purpose of this paper is to present the results of this shallow water experiment and to compare the experimental and numerical data. Also included are results of a preliminary quasi-one-dimensional experiment (depressurization in a straight channel) where we compare the experimental results to those obtained by means of the method of characteristics [13]. This preliminary study showed that the analogy is in particular valid for sub-sonic flows. The largest errors in this analogy are induced by a viscous boundary layer which arises at the bottom of the water bed. Overall, however, the comparison shows very satisfactory agreement. The qualitative flow behavior can be extrapolated to the real reactor situation.

\section{The hydraulic analogy}

\subsection{Fundamentals}

Under conditions, which will be discussed later, the two-dimensional transient mass and moment equations for compressible ideal gas flow and for shallow water flow in a plane bed with vertical boundary walls under gravity $g$ are analogous. The corresponding quantities are shown in table 1.

This analogy is valid if $[10-13]$ :

a) the flow is frictionless in both the gas and the water,

b) the isentropic expansion coefficient $\kappa$ in $\left(p / p_{0}\right)=$ $\left(\rho / \rho_{0}\right)^{\kappa}$ is equal to two for the gas,

c) the water surface height $h$ is small compared to the characteristic length scales $\lambda$ of the surface waves. Otherwise the velocity $a$ of small disturbances would become dependent of $\lambda$ (dispersion),
Table 1

Corresponding quantities for compressible ideal gas flow and for shallow water flow

\begin{tabular}{lll} 
ideal gas & shallow water \\
\hline$\rho \quad=$ density & $h$ & $=$ water surface height \\
$u, v=$ velocity & $u, v$ & $=$ velocity \\
$t \quad=$ time & $t$ & $=$ time \\
$p \quad=$ pressure & $\frac{1}{2} g h^{2}$ & $=-$ \\
$c \quad=$ speed of sound & $a=\sqrt{g h}$ & $=$ speed of small disturbances
\end{tabular}

d) surface tension is negligible if compared to the static water pressure $\rho g h$.

Condition b) restricts the analogy so that we cannot simulate the real reactor flow quantitatively because $\kappa$ is certainly different from two there. If we think of one- and two-phase water as an ideal gas, then the value of $\kappa$ defined as $\kappa \equiv(\rho / p)(\partial p / \partial \rho)_{\text {s }}$ varies between one (two-phase) and 100 ("incompressible" single phase liquid water). This is the reason why we do not compare our experimental results to calculations for the real reactor situation rather than to calculations for the ideal gas.

Conditions a) and d) on the one hand and c) on the other have contradictionary consequences with respect to the water height $h_{0}$ to which the water bed should be filled initially. It was therefore necessary to determine the appropriate height $h_{0}$ in a preliminary experiment where measures for the errors are available. Experiences reported in the literature might not be relevant directly as in the past the hydraulic analogy has been used mainly to study transsonic flow problems where the characteristic length scale $\lambda$ is very small in the resultant shock waves.

\subsection{One-dimensional depressurization model experi- ment}

The ideal gas flow in a pipe as shown in fig. 2 with initial pressure $p_{0}$ after a sudden opening of the right end can easily be computed using the method of characteristics [15]. Therefore, we have set up a similar experiment [13] in a water bed (length $L=1.8 \mathrm{~m}$, width $b=0.4 \mathrm{~m}$ ) and measured the variation of $h$ as a function of time $t$ and position $x$. The experimental procedure is similar to the one described later for the two-dimensional problem. The measurements were made for two values of $h_{0}(20$ and $30 \mathrm{~mm})$. Fig. 3 

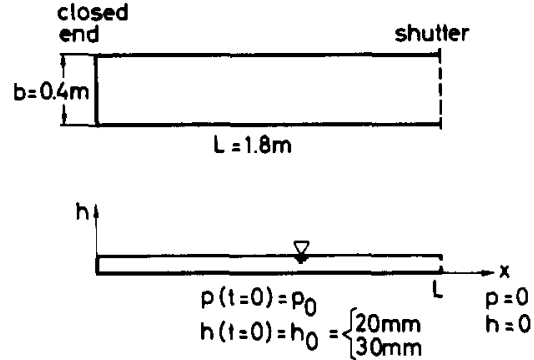

Fig. 2. Geometry of the one-dimensional model.

shows the results in comparison to the computed values.

We observe that the value of $h(L)$ quickly approaches the critical value $h_{0}^{*}=h_{0}\left(\frac{2}{3}\right)^{2}$. This critical value is valid for nonsteady outflow with a velocity equal to the velocity of small disturbances as can be easily deduced from the method of characteristics [15, p. 88].

The height at this position keeps this value until the depressurization wave has been reflected at the closed end $(x=0)$ and returned to the outlet. Then, the water height decreases gradually. No shocks appear. Up to times $3 t^{*}=3 L / \sqrt{g h_{0}}$ the differences between measured and computed heights are nearly independent of $h_{0}$ and typically less than $15 \%$. Later, the differences grow rapidly, in particular for small values of $h_{0}$. The differences are such that the measured values of $h$ are larger than determined theoretically.

This departure can be explained by the build-up of a viscous boundary layer at the bottom of the water bed. A rough estimate of the thickness $\delta$ of the boundary layer can be taken from Prandtl [16] as

$\delta \approx 1.73 \sqrt{v s / w}$

( $s=L-x, \nu=$ viscosity). If $h-\delta$ is taken as the effective water surface height a better agreement is found [13].

In this experiment, the velocity field has been determined too. Similar agreement with the results obtained by the method of characteristics has been found as reported for the height [13].

From this preliminary study, we conclude that $h_{0}$ should be larger than $30 \mathrm{~mm}$. Such large values are possible without violating the condition $h_{0}<<\lambda$ (see above) because the process being investigated here

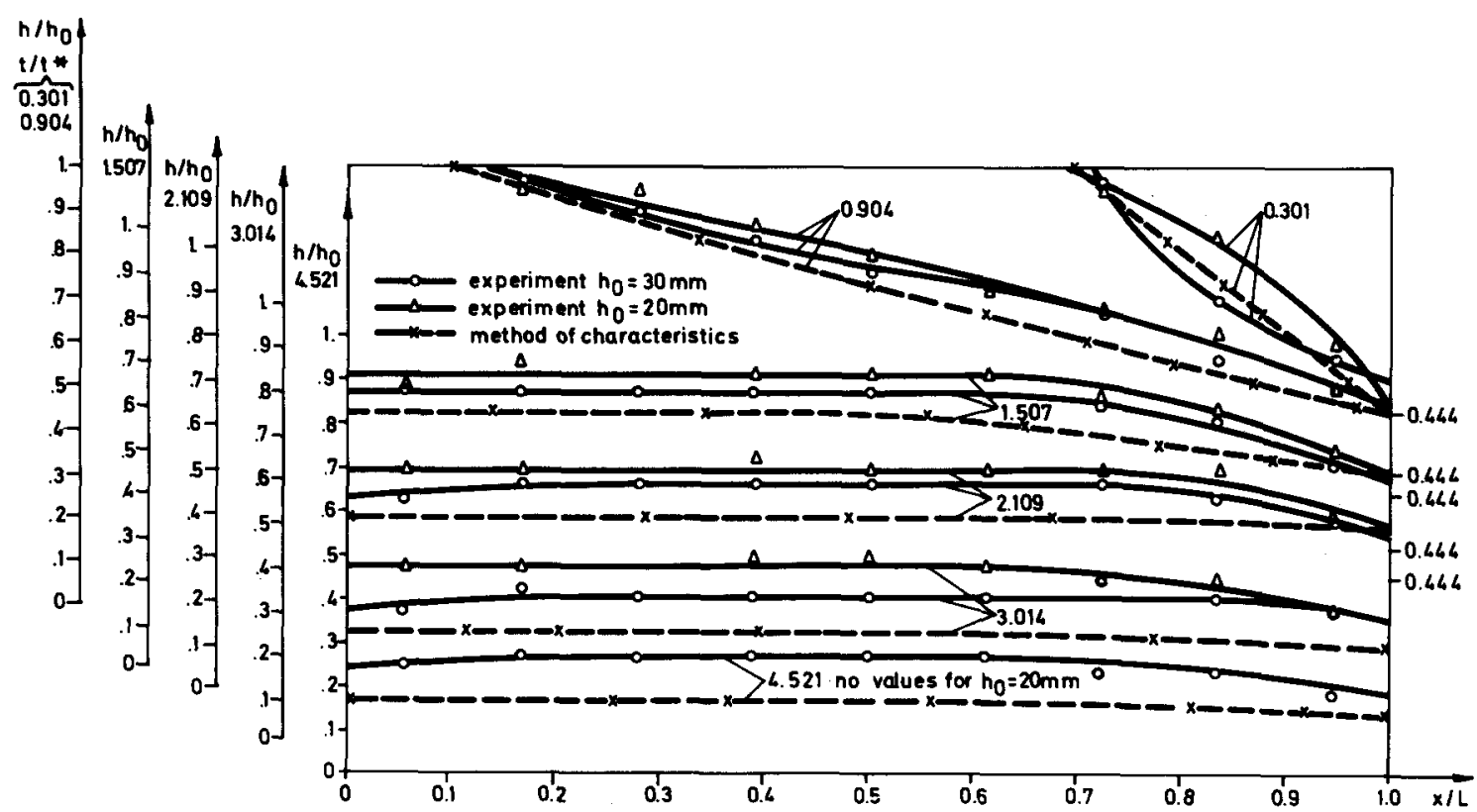

Fig. 3. Water surface height $h$ over initial value $h_{0}$ as a function of space position $x$ for different times $t\left(t *=L / \sqrt{g h_{0}}\right)$. The full curves are for two sets of experiments with different values of $h_{0}$. The dashed curves represent the result obtained by the method of characteristics. 
does not tend to produce steep shock waves but rather smooth pressure transitions.

\section{Experimental setup and procedure}

\subsection{The water-bed}

The size of the water-bed was taken proportional to the HDR downcomer size reduced by a scale factor $k=0.375$. The resultant bed of length $L_{y}=2.839 \mathrm{~m}$ and width $L_{x}=1.744 \mathrm{~m}$, see fig. 4 , was small enough to fit into the laboratory and to be handled without problems but large enough to get sufficient spatial resolution. The bottom (thickness $15 \mathrm{~mm}$ ) and side walls $(10 \mathrm{~mm})$ are made out of plexiglass. The opening at the "broken pipe" location is represented by a gap in the side wall which is closed initially by a shutter. The shutter, once released by hand, is pushed away_by the water load. This mechanism has been found to be optimal in minimizing disturbances to the inside water.

The position of the opening at the side wall (instead of a hole in the middle of the bottom wall) is of advantage as it excludes the tendency of the outflowing water to form the well-known "bath tube" vortex

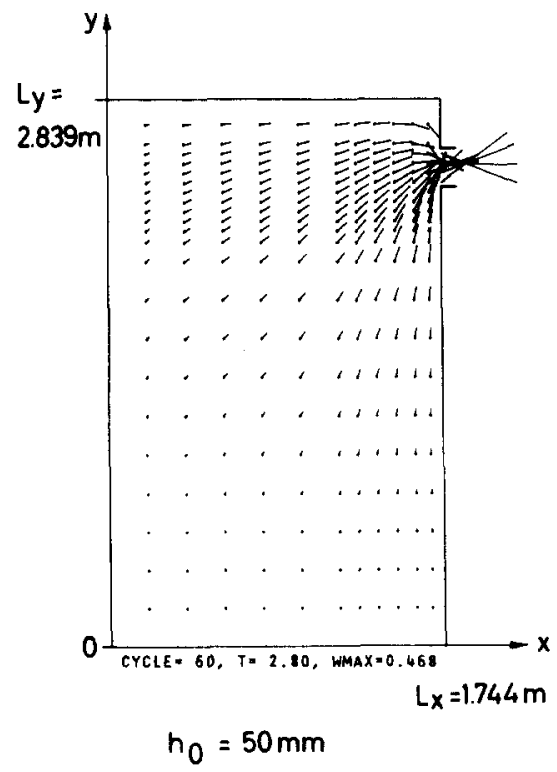

Fig. 4. Vector plot of the computed velocity field for the coarse grid. which would strongly reduce the outflow mass rate. In fact, we have investigated the possibility of reducing the outflow mass rate in a real reactor by a rotation enhancing device. Such a device would reduce the outflow considerably at the late time of blowdown but not so much initially [13].

In the first experiments the size of the gap was scaled to be proportional to the outflow area of the HDR reactor (length of gap $47 \mathrm{~mm}$ ). This gap was found, however, to be too small to give sufficiently large measurable changes in the surface height. The physical reason has to do with the value of $k$ as explained in sec. 7. Also, this small gap requires very small mesh cells and time steps in the computations. Therefore, the geometrical similarity with the HDR was abandoned at the gain of better dynamical similarity by enlarging the gap size to $200 \mathrm{~mm}$.

The water bed is supported by ten beams and adjusted to a horizontal plane within an accuracy of $\pm 1 \mathrm{~mm}$. To allow for a direct reading of the height of the water surface, the transparent side walls are engraved with horizontal lines spaced $1 \mathrm{~mm}$. For better contrast the water is coloured in green using Flourescein (trademark). The surface tension is reduced by adding a standard detergent. The initial water height is chosen as $h_{0}=50 \mathrm{~mm}$, in accordance with the findings of the preliminary experiment and the bigger dimensions of this water bed. The outflowing water $(\approx 250 \mathrm{~kg})$ is gathered in a barrel and refilled after each "blowdown" by a little pump. The time to allow the water to come to rest is at least 5 minutes before "blowdown".

\subsection{Measurement technique}

The height of the water surface as a function of time was registered by means of a movie camera ( 25 frames/s) at 22 distinct positions over a period of $22 \mathrm{~s}$. (For comparison: a small disturbance travels along the long side of the water bed in $t^{*}=L_{y} / \sqrt{g h_{0}}$ $=4.05 \mathrm{~s}$ ). The camera registered a clock which was started simultaneously with release of the shutter at the gap. Later, the height variations were deduced from the films with reference to the engraved height lines. The accuracy of this procedure is $\pm 0.25 \mathrm{~mm}$ in height and $\pm 0.025 \mathrm{~s}$ in time. The smallest height was $37.5 \mathrm{~mm}$.

The fluid surface velocity in the horizontal direc- 
tion inside the water bed was not measured directly rather than deduced from the movement of several cork-pieces ("markers") scattered over the water surface initially. The movement of these markers was registered by a film camera from above the water bed with the time marked by the above mentioned clock. The space positions were measured with reference to a grid of lines (spacing $10 \times 10 \mathrm{~cm}$ ) painted on a glass plate which was put just above the water surface. The upper and lower part of the bed were filmed separately. The films were evaluated later to give the particle positions every second. The accuracy of this measurement is in the order of $\pm 0.5 \mathrm{~cm}$. The resultant positions of the markers were fed into a computer program which computes the velocity by simple finite differences and plots the marker positions at the selected times. For more details see ref. [14].

\section{The computational model}

As mentioned above, we compare the experiments with computations for an ideal gas flow with $\kappa=2$ where the hydraulic analogy is valid.

The finite difference grid is nonequidistant as can be seen from fig. 4 . Two resolutions were used. The "coarse grid" consists of $12 \times 23$ inner grid cells (in $x$ and $y$ ), the "fine grid" of $24 \times 46$ grid cells. The time step was determined dynamically according to linear stability criteria of the code. For the coarse grid $\sim 400$ time steps are required to simulate the flow up to $20 \mathrm{~s}$. No viscous forces are included and free-slip boundary conditions are applied at the side walls.

The outflow boundary condition at the gap was not trivial to define appropriately: It is not appropriate simply to set the water height (density in the code) to zero in the grid cells just outside the opening. This would result in unrealistic supersonic velocities in the neighbourhood of the outflow gap inside the computational domain. The code is not able to compute the critical conditions at the outflow boundary correctly, at least in the present case, where the region downstream the gap is not included in the computational model.

Instead the critical steady state height- (or density-) ratio was used as boundary value $h_{\text {out }}$ :

$h_{\text {out }}=h^{*}=\left(\frac{2}{3}\right) \bar{h}$,

where $\bar{h}$ is the instantaneous spatially averaged height.
This boundary condition still causes errors as it is strictly valid for steady flow only. Initially, the smaller value $h_{0}^{*}=\left(\frac{2}{3}\right)^{2} \bar{h}$, as it was used for the one-dimensional problem, might be more appropriate. However, a distinct characteristic property of the two-dimensional case is the fact that a quasi-steady situation emerges after a short time. In fact, in the straight duct problem we never obtain a steady flow unless the closed end would be replaced by a constant height boundary, e.g. by connecting a large reservoir. In this case, steady state would have been obtained after the depressurization wave has been reflected at this boundary and travelled back to the opening. In two dimensions, the fluid in the immediate neighbourhood of the outlet serves as a reservoir which keeps the water surface height quasi-constant. Due to the size of the gap, which is small compared to the total length of the water bed, the time after which quasi-steady state is created $(\approx 3 \mathrm{~s})$ is so short that the boundary condition $h_{\text {out }}=h^{*}$ gives sufficient accuracy as will be seen from the comparison with the experiment.

\section{Results}

\subsection{Sound and outflow velocity}

In the experiment the velocity $a$ of small disturbances ("sound") was determined by measuring the time from the opening of the shutter after which the first change in height is observable in given distances from the opening. In fig. 5 to 8 the theoretical arrival time of the depressurization wave is marked on the time axis (for further discussion of these figures see below). The wall positions $(x=1.744, y=1.0)$ and

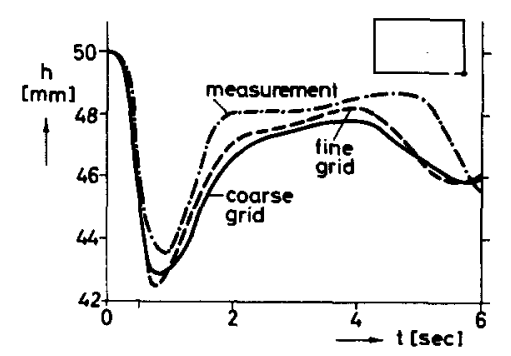

Fig. 5. Water surface height $h(t)$ as a function of time for $(x=1.744, y=2.839)$. The marked theoretical arrival time is $t_{\mathrm{th}}=\mathrm{s} / \sqrt{g h_{0}}$. 


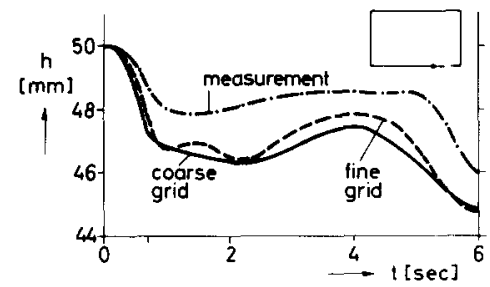

Fig. 6. Same as fig. 5 for $(x=1.744, y=2.0)$.

$(0 ., 1$.$) have been used for five repeated measurements$ of the arrival time. With respect to the velocity of small disturbances we obtain the result $a=0.62 \mathrm{~m} / \mathrm{s}$ $\pm 0.015 \mathrm{~m} / \mathrm{s}$ corresponding to about $2 \%$ statistical relative error. The theoretical value is $\sqrt{g h_{0}}=0.70$ $\mathrm{m} / \mathrm{s}$.

In the computations very small changes in the density are felt shortly after the gap is opened, long before the depressurization reaches the point under consideration. This effect is obviously a result of the iterative scheme. When the depressurization wave reaches the point, the change in density is a bit less abrupt than in the experiment so that the velocity of sound cannot be defined as sharply. But, the resultant estimate for the speed of sound shows no significant departure from the measured value.

The outflow velocity was not measured. The computed value, e.g. $w_{\text {out }}=0.516 \mathrm{~m} / \mathrm{s}$ for the fine grid at $t=4.15 \mathrm{~s}$ (which falls in the quasi-steady period), corresponds within $8 \%$ to the critical outflow velocity $\sqrt{g h_{\text {out }}}=0.560 \mathrm{~m} / \mathrm{s}$ according to steady one-dimensional theoretical predictions.

\subsection{Water-height (density) at the boundaries}

Measured and computed water heights $h(t)$ at different points or $h(y)$ at a specific time near the outlet are shown in figs. 5 to 9 . From these figures we con-

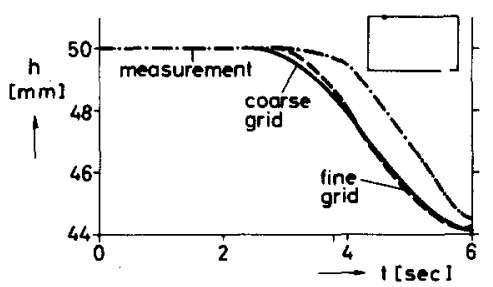

Fig. 7. Same as fig. 5 for $(x=0.0, y=0.5)$.

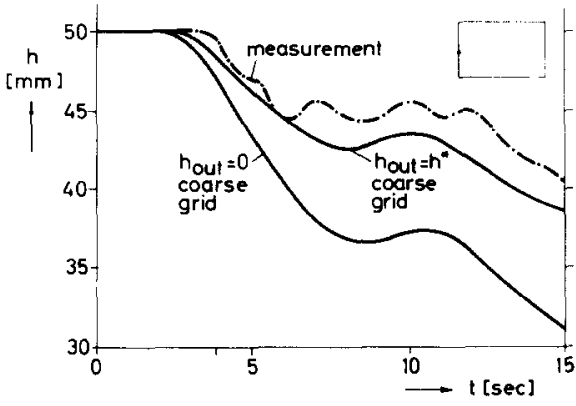

Fig. 8. Same as fig. 5 for $(x=1.0, y=0.0)$.

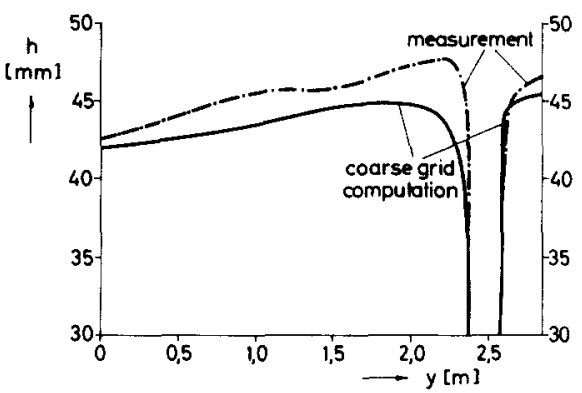

Fig. 9. Water surface height $h(y)$ as a function of space $(x=1.744)$ for $t=8.0 \mathrm{~s}$.
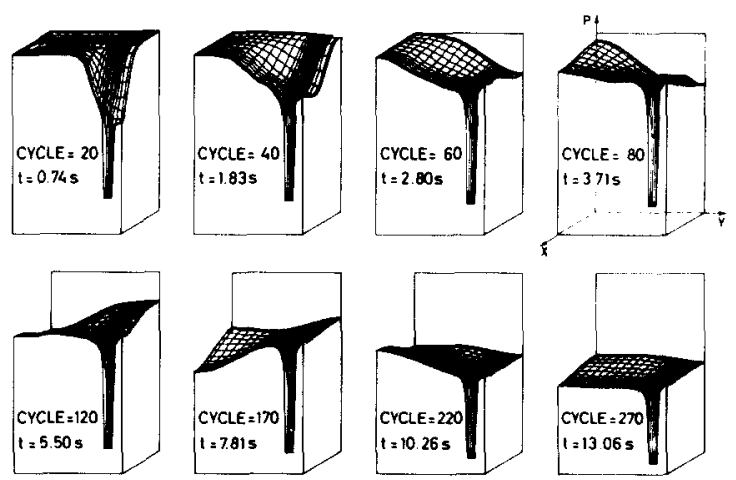

Fig. 10. Perspective plots of the computed pressure field $p(x, y)$ at distinct times $t$ (cycles).

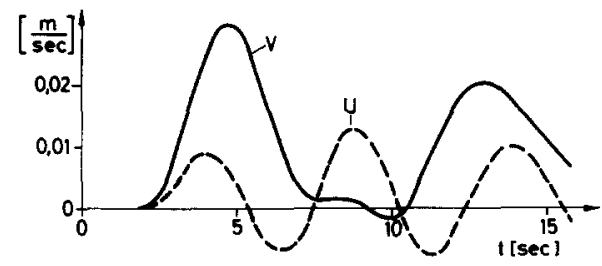

Fig. 11. Computed axial $(v)$ and azimuthal $(u)$ velocity components as a function of time $t$ for $(x=0.6 \mathrm{~m}, y=0.6 \mathrm{~m})$. 
clude that the agreement between experiments and computations with $h_{\text {out }}=h^{*}$ as boundary condition is good. Figs. 5 to 7 demonstrate that the computational results are sufficiently insensitive to the grid resolution. Fig. 8 includes results for the boundary condition $h_{\text {out }}=0$, which, as expected, gives too rapid height reductions. From these figures we again find that the velocity of small disturbances is lower in the experiment than in the computations. The temporal (figs. 5-8) and spatial (fig. 9) height variations are slightly weaker in the computations than in the experiment which indicates that the numerical method tends to dampen the high frequency field components more than the lower ones. This effect is known as numerical damping.

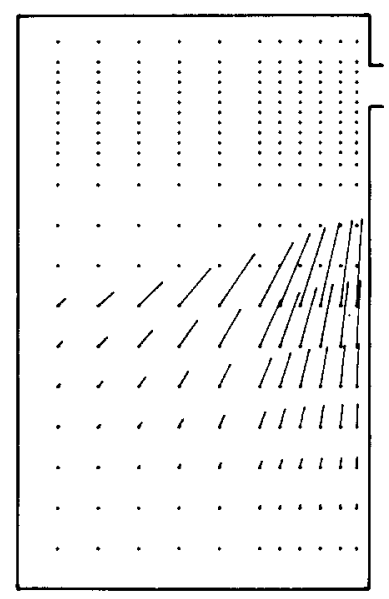

CYCLE $=50, T=2.32, \operatorname{man} x=0.040$
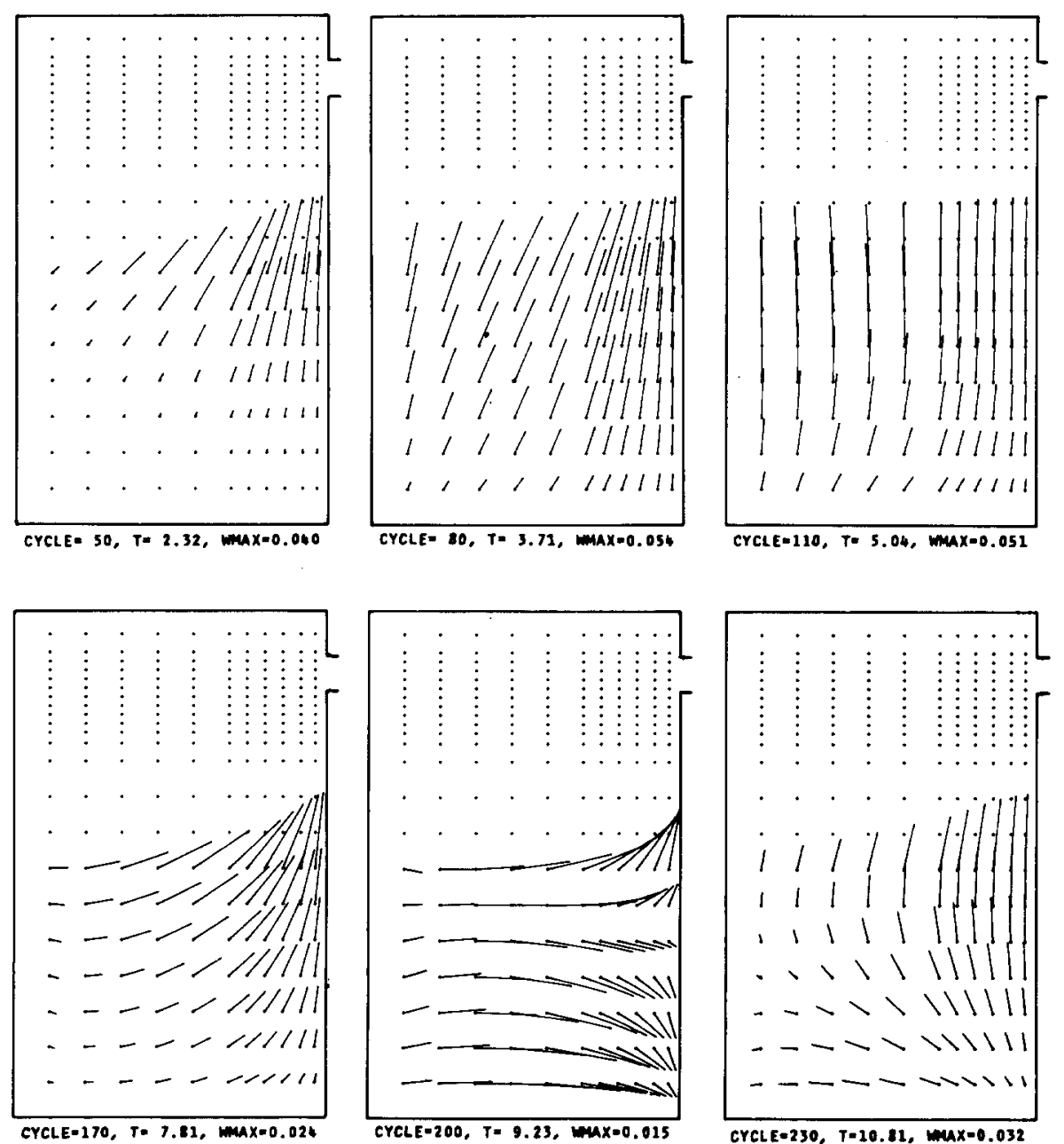

CYCLE $=110, T=5.04, \max =0.051$

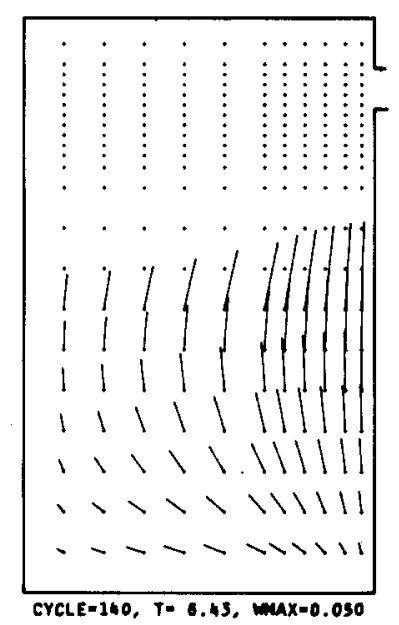

\subsection{Pressure-distribution inside the domain}

A sequence of computed pressure fields for different times is plotted perspectively in fig. 10 which gives a good impression of the qualitative behaviour of the pressure field in the downcomer. For cycle 20 we see the expected pressure drop near the opened nozzle. Shortly thereafter, however, the pressure recovers in the surroundings of the nozzle to about the original value, although the depressurization wave has not yet reached all boundaries, which is typical for the two-dimensional converging flow situation as explained in sec. 4 . Thereafter, the outflow is quasi-static. At cycle 60 we observe a reflection at the wall opposite to the nozzle. In reality this

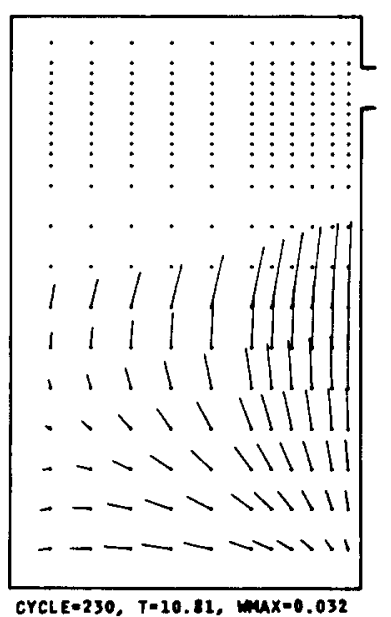

Fig. 12. Computed velocities in the lower half of the downcomer for a sequence of times. 
reflection is the superposition of the two waves coming around the two sides of the downcomer. At cycle 80 the wave has been reflected at the lower end $(y=0)$. (This reflection has no analogy in the real reactor situation where a constant pressure boundary condition would be more appropriate. This boundary condition is, however, consistent with the present experiment.) From then on, the fluid slopes back and forth around the downcomer as well as up and down. The period of these oscillations in each direction is proportional to the geometrical lengths over the speed of small disturbances. Gradually the mean pressure value decreases due to the outflowing fluid.

The same behaviour has been observed in the water
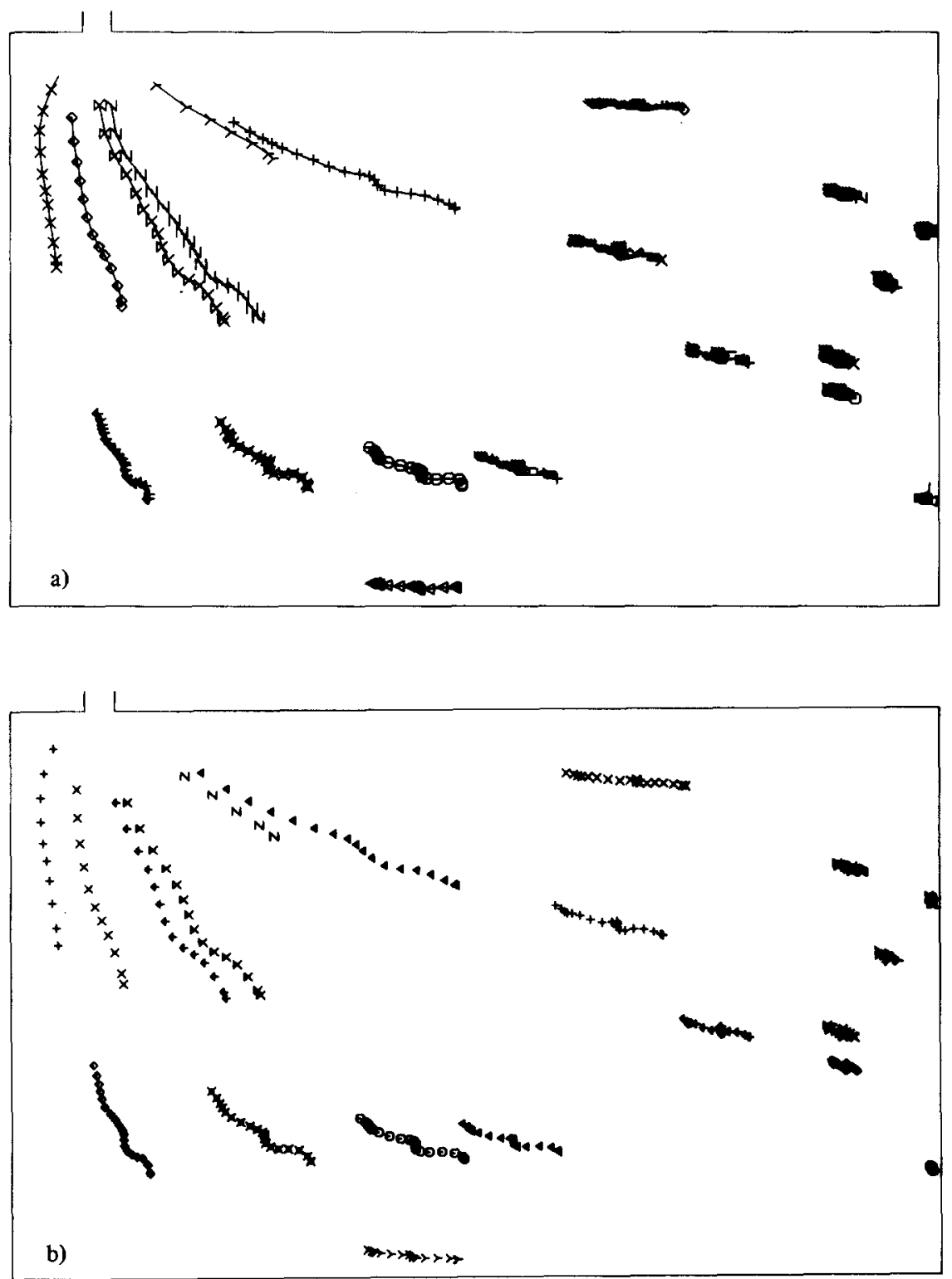

Fig. 13. Trajectories of 20 markers a) measured, b) computed results. 
bed (documented on the movie films). As it is not feasible to measure the surface height at each point inside the domain, similar figures cannot be shown for the experiment. Qualitatively similar figures for real reactor situations have been presented in $[1,2]$. However, the azimuthal oscillations are more pronounced in the present study which is partly a consequence of the different boundary condition at the lower end of the downcomer and partly a consequence of the different equation of state.

\subsection{Velocity field}

The velocity components $u$ and $v$ ( $x$ - and $y$-components) as a function of time (at an arbitrarily chosen site) is shown in fig. 11 as computed. The $u$-component shows the characteristic azimuthal oscillations, whereas the $v$-component is oscillating without much becoming negative. Its mean value corresponds to the mean outflow. The spatial variation of the velocity field is illustrated by fig. 4 . This is a vector plot with vectors shown in proportion to the magnitude and direction of the computed velocity vectors. Because of the large magnitude of the velocities near the outlet, the vectors in the lower part of the downcomer can hardly be seen in this type of figure. Therefore, better insight is obtained by producing such vector plots for the upper and lower part of the domain separately. Fig. 12 shows a sequence of such plots for the lower part, which clearly resembles the oscillations of the fluid as deduced already from the pres. sure profiles.

\subsection{Marker trajectories}

Inside the water bed it is easiest to track the markers floating with the fluid instead of measuring velocities. Fig. 13 shows the result. Fig. 13a depicts the measured positions of 20 particles at 22 sub. sequent times with intervals of $1 \mathrm{~s}$. For each marker these positions are connected by straight lines to give the trajectory. For situations where the fluid stagnates several subsequent marker positions coincide on nearly the same point so that the details can no longer be separated.

The same type of figure, fig. 13b, has been produced by tracking virtual markers in the numerical simulation: the same initial positions are used and the position is moved at each time step in a Lagrangian manner according to the surrounding velocity field. As can be seen from fig. 13 the computed trajectories compare extremely well with the experimental results.

\section{Error analysis}

In spite of the rather small variations of the water surface height in the experiments, the errors caused by the measurement technique and experimental scatter are of minor importance and generally amount to less than a few percent.

The main departure between experimental and numerical results for the two-dimensional case has been found with respect to the velocity of small disturbances. Whereas this velocity is slightly overestimated by the numerical model due to the implicit scheme, it is smaller by about $10 \%$ in the experiments. No convincing explanation has been found for this discrepancy. It seems to be a consequence of the two-dimensionality, as in the one-dimensional case the measured velocity of "sound" agrees well with the result obtained from the method of characteristics (see fig. 3). Some later differences in the computed and measured velocities (in particular in the onedimensional case) are explainable by the build-up of a viscous boundary layer. Other errors like those effected by surface tension and dispersion appear to be negligible. This is attributable to the sufficient size of the water bed and the fact that shock waves are absent. Overall, the hydraulic analogy has been found to allow for sufficiently accurate experiments on twodimensional gas-flow problems.

In the computations, besides the small errors introduced by the implicit scheme requiring pressure iterations, errors are caused by the finite grid spacings and the boundary condition at the outlet. However, both have been shown to be reasonably small.

\section{Conclusions}

We have reported results of an experimental and numerical study of a model for the flow in a nuclear reactor downcomer after a sudden break of one of the primary coolant circuits immediately at the vessel. 
The relevance of the present study with respect to reality is of qualitative nature. It resembles the following features:

- The depressurization starts with an initial phase where the pressure drops but the fluid is still at rest. The form of the depressurization wave is rather smooth which is a consequence of the geometrical expansion and the strong dependence of the speed of sound on the density (surface height).

- Then the fluid is accelerated and the pressure recovers near the outlet. This acceleration phase is not dependent of the speed of sound. It is known to appear in incompressible fluid as well [17].

- After the fluid has been accelerated and the depressurization wave has been reflected at all boundaries a quasi-static flow situation emerges where the outflow is nearly constant and the fluid oscillates up and down and around the downcomer. These fluid oscillations cause the biggest loads on the core barrel [3]. The mean pressure value decreases monotonically according to the outflow.

The main differences to reality result from the equation of state for which a very specific form (ideal gas, $\kappa=2$ ) had to be used here in order to make the hydraulic analogy applicable. For a larger $\kappa$ the pressure is much more sensitive to small density changes. This explains why almost no measurable effect was seen in the model-experiment with a gap directly proportional to the assumed break area in reality. If $k$ is smaller, the velocity of sound becomes less dependent on the density (in isentropic flow $a / a_{0}=\left(\rho / \rho_{0}\right)^{(k-1) / 2}$ ) which increases the steepness of the depressurization wave.

From the comparison we conclude that YAQUIR is well suited for the analysis of the depressurization of the downcomer within the geometrical model used here. It is an open question whether it would be able to predict the critical conditions at the outlet if the outer region is included in the computational domain.

We suggest that the presented results shall be used as a standard test problem for two-dimensional compressible flow analysis techniques.

\section{Nomenclature}

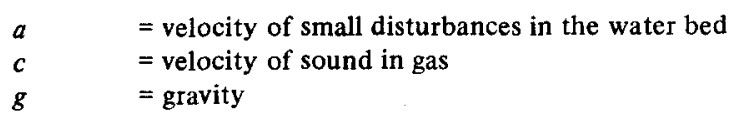

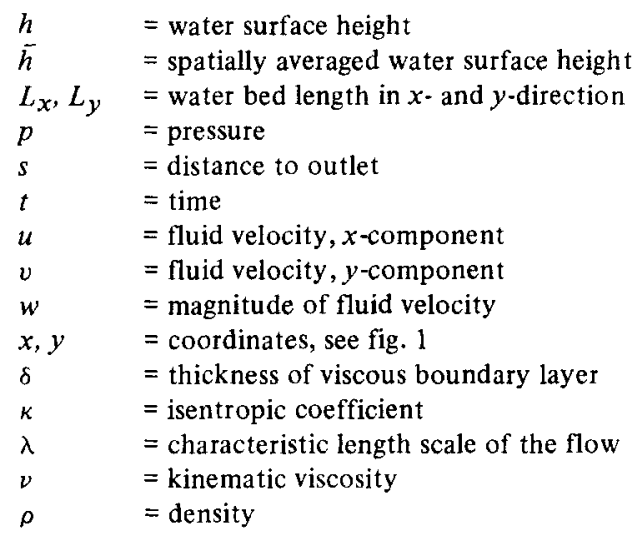

\section{Subscripts}

$\begin{array}{ll}0 & =\text { initial } \\ \text { out } & =\text { outlet } \\ \mathrm{S} & =\text { isentropic }\end{array}$

\section{References}

[1] R. Krieg, E.G. Schlechtendahl, K.-H. Scholl, Nucl. Eng. Des. 43 (1977) 419.

[2] R. Krieg, E.G. Schlechtendahl, K.-H. Scholl, U. Schumann, in: 4 th Int. Conf. on Structural Mech. in Reactor Techn., San Francisco (1977), paper B5/1.

[3] F. Katz, R. Krieg, A. Ludwig, E.G. Schlechtendahl, K. Stölting, in: 4 th Int. Conf. on Structural Mech. in Reactor Techn., San Francisco (1977), paper B5/2.

[4] C.W. Hirt, A.A. Amsden, J.L. Cook, J. Comp. Phys. 14 (1974) 227.

[5] T. Grillenberger, MRR-P-24 (1976).

[6] S. Fabic, Kaiser Engineers Report No. 67/49/R (1967).

[7] G.A. Hughes, KWU-Arbeitsbericht 38/74, R 112 (1974).

[8] P. Pana, M. Müller, Nucl. Eng. Des. 36 (1976) 183.

[9] J.K. Dienes, C.W. Hirt \& L.R. Stein, LA-NUREG-6772MS (1977).

[10] D.P. Riabouchinsky, Compt. Rend. de l'Academie des Sciences 195 (1932) 22, p. 998.

[11] E. Preiswerk, Dissertation, ETH Zürich, Institut für Aerodynamik, Mitteilung No. 7, (1938).

[12] H. Bömelburg, Mitteilungen aus dem Max-Planck-Institủt für Strömungsforschung, Göttingen, Nr. 10 (1954).

[13] F. Kedziur, Diplomarbeit, Universität Karlsruhe, Institut für Reaktortechnik (1976).

[14] N. Moussiopoulos, Studienarbeit, Universität Karlsruhe, Institut für Reaktortechnik (1977).

[15] J. Zierep, Vorlesungen über theoretische Gasdynamik, (G. Braun, Karlsruhe, 1963).

[16] L. Prandt, Führer durch die Strömungslehre, (F. Vieweg \& Sohn, Braunschweig, 6. Aufl., 1965) p. 163.

[17] U. Schumann, KFK 2324 (1976). 